\title{
Cytokine Storm in COVID-19 in Diabetic Patients: A Review
}

\author{
Arpita Mazumder ${ }^{1}$, Nilanjan Roy ${ }^{2}$ \\ ${ }^{1}$ Undergraduate Medical Student (MBBS), Mymensingh Medical College, Mymensingh, Bangladesh; ${ }^{2}$ Graduate (B.Sc. in \\ Biomedical Engineering), Department of Biomedical Engineering, Military Institute of Science and Technology, Dhaka, Bangladesh
}

[Received: 17 August 2020; Accepted: 28 September 2020]

\begin{abstract}
COVID-19, a global pandemic has created a worldwide disaster since its first outbreak in December 2019 in Wuhan, China. Studies based in China have reported that people with diabetes and infected with COVID-19 showed higher hospital admission rates, serious pneumonia development, and higher mortality rates. SARS-CoV-2 can increase the metabolism of glucose by fueling immune cell overproduction - a phenomenon known as a 'cytokine storm'. Glucose metabolism is said to contribute to diverse COVID-19 outcomes. In this study, we are overviewing the linkage among SARS-CoV-2, diabetes, and cytokine storm for a better understanding of aggravating health conditions in diabetic patients due to this SARS$\mathrm{CoV}-2$ virus. In conclusion, this review will contribute to understanding complications that is rising among diabetic patients due to SARS-CoV-2 induced cytokine storm. [Bangladesh Journal of Infectious Diseases, October 2020;7(suppl_2):S46-S49]

Keywords: COVID-19; diabetes; cytokine storm

Correspondence: Nilanjan Roy, Biomedical Engineer, Department of Biomedical Engineering, Military Institute of Science and Technology, Dhaka, Bangladesh; Email: nilanjan.roy0@ gmail.com; Cell no.: +8801775022326

Conflict of interest: Authors declare that there is no conflict of interest.

Funding agency: The authors did not receive any type of funding for this study.

Contribution to authors: Mazumder A, Roy N involved in literature search up to manuscript writing and revision.

How to cite this article: Mazumder A, Roy N. Cytokine Storm in COVID-19 in Diabetic Patients: A Review. Bangladesh J Infect Dis 2020;7(suppl_2):S46-S49

Copyright: @2020. Mazumder and Roy. Published by Bangladesh Journal of Infectious Diseases. This article is published under the Creative Commons CC BY-NC License (https://creativecommons.org/licenses/by-nc/4.0/). This license permits use, distribution and reproduction in any medium, provided the original work is properly cited, and is not used for commercial purposes.
\end{abstract}

\section{Introduction}

SARS-CoV-2 is a new strain of coronavirus that has taken the world by storm. The high spread rate of the SARS-CoV-2 virus has caused an enormous amount of deaths and made a huge negative impact on our economy and health systems ${ }^{1}$. Patients with SARS-CoV-2 infection can show a wide range of symptoms from mild to severe. The most common symptoms include fever, cough, and shortness of breath. Patients with acute respiratory distress syndrome will get worse quickly and die from multiple organ failure ${ }^{2}$. Currently, the exact transmission mechanism of SARS-CoV-2 is not yet fully known. The major routes of transmission within a susceptible population are human-tohuman transmission through droplets and oral$\mathrm{fecal}^{1}$. The median incubation time of SARS-CoV-2 has been reported to be 5.1 days and 97.5 percent of infected subjects will experience symptoms within 
11.5 days of infection. Based on those figures, it can be concluded that after 14 days of observation or quarantine, 101 out of 10,000 cases will experience symptoms ${ }^{3}$. Confirmation of cases of suspected infection of SARS-CoV-2 is conducted by identifying specific viral sequences with nucleic acid amplification tests such as real-time reverse PCR. So far, no specific vaccine or medicine is available to treat this deadly virus. Another global disease is diabetes that is estimated to affect 463 million adults (20-79 years) in 2019, representing 9.3 percent of this age group's world population ${ }^{4}$. It has been seen that diabetic patients infected with SARS-CoV-2 are more likely to die or have critical health conditions and they are needed to be hospitalized quickly. Reports suggest that this the consequence of cytokine storm that is developed within diabetic patients diagnosed with COVID-19. By reviewing literatures, this study aims to clarify the cytokine storm phenomenon in this regard.

\section{How Does Cytokine Storm Work in COVID-19?}

The immune system has a strong sense of distinguishing what is self and what is non-self. It kills foreign pathogens or any other dangerous molecules that come in its way. Unfortunately, sometimes the immune system commences an exaggerated response and become so hyperactive that instead of killing non-self, it starts to kill the self-antigens. Cytokine storm is an example of a hyperactive immune response. Cytokine is a small protein that acts as a signaling molecule between the immune system cells for conveying signals (mostly when the foreign invaders attack) from one immune system cells to another. It's termed as "storm" since the immune system cells being hyperactive and release large amounts of cytokines. In the case of SARS (Severe Acute Respiratory Syndrome) and MERS (Middle East Respiratory Syndrome) studies show, the evidence of increased circulating levels of pro-inflammatory cytokines (e.g., Interferon $\gamma$, Interleukin, IL-1 $\beta$, IL-6, IL-12) and Chemokines (CXCL10, and CCL2) which were responsible for severe pulmonary inflammation ${ }^{5}$.

About COVID-19, it has been reported that infected patients show high levels of pro-inflammatory cytokines ${ }^{6}$. This theory comes from when the people who require ICU admission and their blood picture reveals a higher concentration of CXCL10, CCL2, and Tumor necrosis factor-alpha (TNF $\alpha$ ) than those who didn't require ICU admission. The rising levels of IL-1B, IFN $\gamma$, CXCL10, and CCL2 suggests activation of T-helper $1(\mathrm{Th}-1)^{7}$. What is surprising here, in SARS-CoV 2, IL-4, and IL-10 releases from $\mathrm{T}$-helper-2 (Th-2) cells that are known to suppress the inflammation and it's not happened in $\mathrm{SARS}^{8}$. Therefore, when the cytokine storm gets the upper hand, the plasma and the inflammatory cells extravasate into the pulmonary cells and due to fluid, there is edema and the inflammation in the air sacs which limits the ability of a person to breathe properly. In China, doctors declared $81.0 \%$ of COVID-19 symptoms are mild and according to WHO, a patient who will get severe pneumonia will be considered as a severe category. A study found that $17 \%$ of patients develop Acute Respiratory Distress Syndrome (ARDS) and among them, 65\% deteriorate rapidly due to multi-organ failure ${ }^{9}$. Another study shows, ARDS is greatly associated with who are immunocompromised like a diabetic patient, hypertensive patient, and the geriatric age group $(>65 \text { years })^{10}$. Besides the pulmonary symptoms, while the blood vessels due to loss of fluid start to become more and more constrict consequently, the gaseous exchange collapses, and all other organs are directed towards the ultimate failure. The heart failure, renal failure, liver injury, GIT symptoms all mainly are the results of cytokine storm induced ARDS.

\section{Why Cytokine Storm Is More Dangerous for Diabetic Patients With COVID-19?}

A Chinese meta-analysis data which presented a report by experimenting on 1527 patients showed the most common comorbidities along with COVID-19 are hypertension $(17.1 \%, 95 \%$ CI 9.9 $24.4 \%)$, cardio-cerebrovascular disease (16.4\%, 95\% CI $6.6-26.1 \%)$, then diabetes $(9.7 \%, 95 \%$ CI $6.9-12.5 \%)^{11}$. It's been recorded that, the diabetic patient diagnosed with COVID-19 had more IL-6 and C-reactive protein (CRP) than those who don't have diabetes ${ }^{12}$. Furthermore, Diabetic patients who have been diagnosed with COVID-19 are showing high ICU admission and higher mortality rate mostly caused by pulmonary infections ${ }^{13,} 14,15$. These overwhelming pulmonary infections are mainly the result of the cytokine storm. Cytokine storm is severe in patients who are diabetic and also have been diagnosed with COVID-19. Among diabetic patients, more vulnerable people are those who have uncontrolled diabetes mellitus. Because hyperglycemia stimulates the synthesis and release of cytokines ${ }^{16}$. A point to be noted, the pancreas possesses ACE-2 (Angiotensin Converting Enzyme-2) enzyme and SARS- CoV-2 enter into the host cell by binding to the ACE-2 through their glycoprotein spike in the capsule to the pancreatic beta cells and damage the capability to release insulin $^{17}$. This raises the diabetic patient's blood glucose level and therefore results in acute 
hyperglycemia. This deteriorates the glycemic status in COVID-19 patients and so it may overwhelm the cytokine level and its effects. From diabetes pathophysiology it's been known, diabetic patients have decreased the number of Angiotensin Converting Enzyme (ACE-2) which acts as antiinflammatory and anti-oxidant protein. ACE-2 can be found in the lungs, kidneys, vascular system, and intestinal endothelium ${ }^{18}$. Since the depletion of enzymes in diabetes, it increases the risk of severe lung injury and also acute respiratory distress syndrome $(\mathrm{ARDS})^{19}$. COVID-19 binds to this ACE2 when it enters into the host cell and It aggravates the lung injury ${ }^{20}$.

\section{Discussion}

COVID-19 and diabetes are a deadly duo as patients having both of these are likely to have serious complications and die from the virus due to high blood sugar as it weakens the immune system and ultimately reducing its ability to fight infections. Although cytokine storm is a determinant factor to describe high death rates of diabetic patients infected with COVID-19, there are still some unanswered questions regarding factors like well-controlled diabetes, the effect of obesity, beta-cell dysfunction/destruction, glycaemic deterioration, ethnicity-specific factors, etc. which are needed to be clarified. As there is still no vaccine for COVID-19 in our hands, strict COVID19 diabetes plans and protocols should be followed. This includes eating healthy carbs, taking prescribed insulin and other medications timely, having extra glucagon and ketone strips at home, drinking plenty of fluids, and checking blood sugar regularly. Preparation and precautions are the best protection for diabetic patients in this time of the global pandemic.

\section{Conclusion}

One of the major comorbidities associated with COVID-19 mortality is diabetes. Several studies have attempted to understand the potential vulnerability of diabetic patients to SARS-CoV-2 infection. The hyperimmune response called cytokine storm has been also found to play a major role in sudden deterioration in SARS-CoV-2 infected individuals with comorbidities including diabetes. Therefore, these patients with diabetes, infected with SARS-CoV-2 have been reported to have elevated baseline cytokine levels, such as IL$1 \beta$ and IL-6. Additionally, persistent low-grade inflammation tends to further aggravate the immune response and lead it to hyperimmune response (cytokine storm). Hence, both diabetes and cytokine storm are complementary to each other and help to worsen the conditions of diabetic patients infected with the SARS-CoV-2 virus. Based on the available evidence, it is recommended that diabetic patients must follow a healthy lifestyle and strict control of blood glucose, HTN, and lipids to decrease the risk of severity that might occur due to SARS-CoV-2 viral infection.

\section{References}

1. Ciotti M, Angeletti S, Minieri M, Giovannetti M, Benvenuto D, Pascarella S et al. COVID-19 Outbreak: An Overview. Chemotherapy. 2019;64(5-6):215-223

2. Wang D, Hu B, Hu C, Zhu F, Liu X, Zhang J et al. Clinical Characteristics of 138 Hospitalized Patients With 2019 Novel Coronavirus-Infected Pneumonia in Wuhan, China. JAMA. 2020;323(11):1061

3. Lauer S, Grantz K, Bi Q, Jones F, Zheng Q, Meredith H et al. The Incubation Period of Coronavirus Disease 2019 (COVID-19) From Publicly Reported Confirmed Cases: Estimation and Application. Annals of Internal Medicine. 2020;172(9):577-582

4. Saeedi P, Salpea P, Karuranga S, Petersohn I, Malanda B, Gregg E et al. Mortality attributable to diabetes in 20-79 years old adults, 2019 estimates: Results from the International Diabetes Federation Diabetes Atlas, 9th edition. Diabetes Research and Clinical Practice. 2020;162:108086

5. Channappanavar R, Perlman S. Pathogenic human coronavirus infections: causes and consequences of cytokine storm and immunopathology. Seminars in Immunopathology. 2017;39(5):529-539

6. Huang C, Wang Y, Li X, Ren L, Zhao J, Hu Y et al. Clinical features of patients infected with 2019 novel coronavirus in Wuhan, China. The Lancet. 2020;395(10223):497-506

7. Coperchini F, Chiovato L, Croce L, Magri F, Rotondi M. The cytokine storm in COVID-19: An overview of the involvement of the chemokine/chemokine-receptor system. Cytokine \& Growth Factor Reviews. 2020;53:25-32

8. Zhang C, Wu Z, Li J, Zhao H, Wang G. Cytokine release syndrome in severe COVID-19: interleukin-6 receptor antagonist tocilizumab may be the key to reduce mortality. International Journal of Antimicrobial Agents. 2020;55(5): 105954

9. Chen J, Qi T, Liu L, Ling Y, Qian Z, Li T et al. Clinical progression of patients with COVID-19 in Shanghai, China. Journal of Infection. 2020;80(5):e1-e6

10. Wu C, Chen X, Cai Y, Xia J, Zhou X, Xu S, et al. Risk Factors Associated With Acute Respiratory Distress Syndrome and Death in Patients With Coronavirus Disease 2019 Pneumonia in Wuhan, China. JAMA Internal Medicine. 2020;180(7):934

11. Li B, Yang J, Zhao F, Zhi L, Wang X, Liu L et al. Prevalence and impact of cardiovascular metabolic diseases on COVID-19 in China. Clinical Research in Cardiology. 2020;109(5):531-538

12. Guo W, Li M, Dong Y, Zhou H, Zhang Z, Tian C et al. Diabetes is a risk factor for the progression and prognosis of COVID -19. Diabetes/Metabolism Research and Reviews. 2020;36(7)

13. Yang X, Yu Y, Xu J, Shu H, Xia J, Liu H, et al. Clinical course and outcomes of critically ill patients with SARS-CoV-2 pneumonia in Wuhan, China: a single-centered, retrospective, observational study. Lancet Respiratory Medicine 2020;8(5):475-481 
14. Zhang J, Dong X, Cao Y, Yuan Y, Yang Y, Yan Y et al. Clinical characteristics of 140 patients infected with SARS-CoV-2 in Wuhan, China. Allergy. 2020;75(7):17301741

15. Guan W, Ni Z, Hu Y, Liang W, Ou C, He J et al. Clinical Characteristics of Coronavirus Disease 2019 in China. New England Journal of Medicine. 2020;382(18):1708-1720

16. Jafar N, Edriss H, Nugent K. The Effect of Short-Term Hyperglycemia on the Innate Immune System. The American Journal of the Medical Sciences. 2016;351(2):201-211

17. Yang J, Lin S, Ji X, Guo L. Binding of SARS coronavirus to its receptor damages islets and causes acute diabetes. Acta Diabetologica. 2009;47(3):193-199
18. Cuschieri S, Grech S. COVID-19 and diabetes: The why, the what and the how. Journal of Diabetes and its Complications. 2020;34(9):107637

19. Pal R, Bhansali A. COVID-19, diabetes mellitus and ACE2: The conundrum. Diabetes Research and Clinical Practice. 2020;162:108132

20. Hoffmann M, Kleine-Weber H, Schroeder S, Krüger N, Herrler T, Erichsen S et al. SARS-CoV-2 Cell Entry Depends on ACE2 and TMPRSS 2 and Is Blocked by a Clinically Proven Protease Inhibitor. Cell. 2020;181(2):271-280.e8 